\title{
CONOCIMIENTOS ALIMENTARIO-NUTRICIONALES Y \\ ESTADO NUTRICIONAL DE ESTUDIANTES DE CUARTO \\ AÑO BÁSICO SEGÚN ESTABLECIMIENTOS PARTICULARES \\ Y SUBVENCIONADOS DE LA CIUDAD DE TALCA
}

\author{
KNOWLEDGE OF FOOD AND NUTRITION \\ STATUS OF STUDENTS OF 4TH GRADE IN PRIVATE \\ AND SEMI-PRIVATE SCHOOLS OF CITY OF TALCA
}

\author{
José Luis Pino V., Miguel Ángel López E., María Isabel Cofré T., \\ Caroll González R., Liliana Reyes C.
}

Escuela de Nutrición y Dietética, Universidad del Mar, sede Centro Sur. Talca.

\begin{abstract}
The malnutrition by excess of school age children is a problem that may also be treated at school, because Children spend most of their time at school. It was studied knowledge about food of students from year four in primary school of private and semi-private schools of Talca city. An instrument was created and was applied to measure the knowledge of eating habits in children in a sample of 273 students. The average percentage of knowledge that students have, was $61,4 \pm 14 ; 75 \%$. Students from private schools had a better domain in the knowledge of eating habits $(p<0.005)$. Insufficient knowledge can effect the nutritional status of children. This research must be confronted from a multifactorial perspective, even though, only knowledge does not indicate enough success in the nutritional recovery of the children.
\end{abstract}

Keywords: Knowledge, food habits, school fourth grade, well-nourished, school grant.

Este trabajo fue recibido el 4 de Marzo de 2010 y aceptado para ser publicado el 12 de Septiembre de 2010.

\section{INTRODUCCIÓN}

A nivel nacional, la malnutrición por exceso se ha convertido en un considerable problema de salud pública a través de todo el ciclo vital, en el cual la población infantil constituye un porcentaje importante dentro del país, según los últimos datos existe una cifra cercana al $21 \%$ de obesidad (1-4).

El continuo aumento en las cifras de malnutrición por exceso en la etapa escolar se ha asociado a los estilos de vida que lleva actualmente esta población, caracterizados al igual que en el adulto, por sedentarismo con un gran número de horas frente al televisor, y hábitos alimentarios poco apropiados, con bajo consumo de verduras, frutas y lácteos, y un alto consumo de alimentos procesados de alta densidad energética.

La alta cobertura del sistema escolar chileno ofrece una excelente oportunidad para la educación alimentaria y nutricional (5). Conjuntamente, la alimentación del niño en la etapa escolar debe estar basada en una ingesta variada que asegure un buen estado nutricional, fomente la instauración de hábitos alimentarios correctos y perdurables (6).

La FAO y la OMS, considerando que los factores de riesgo de las enfermedades crónicas del adulto se inician en etapas tempranas de la vida, han hecho un llamado a efectuar acciones de prevención en la niñez y juventud, tendientes a fomentar hábitos de vida saludables y destacan especialmente el rol que juegan las escuelas, identificándolas como centros ideales de promoción de la salud comunitaria (7).

Las intervenciones a nivel escolar constituyen una de las estrategias más importantes para enfrentar la obesidad en la población infantil, ya que la cobertura escolar es prácticamente de un $100 \%$ en los primeros niveles de enseñanza básica. Así mismo, dichos niveles conforman un período de desarrollo en hábitos de ali- 
mentación y actividad física, ya que el contacto con los profesores es continuo. Aún cuando está demostrado que para la prevención de la obesidad es necesario lograr cambios conductuales en los aspectos de alimentación y actividad física, el primer paso necesariamente debe ser la adquisición de un nivel de conocimientos básicos en estos aspectos $(8,9)$.

Los niños están la mayor parte del día en la escuela, especialmente los que asisten a establecimientos de jornada completa. Desde el punto de vista nutricional, no sólo tienen oportunidad de ingerir alimentos para satisfacer sus necesidades biológicas, tanto en el desayuno, las colaciones, el almuerzo o la cena, sino también una dimensión social donde comparten, adquieren e imitan hábitos de alimentación de sus pares (10).

La infancia es una etapa fundamental en la obtención y desarrollo de hábitos y pautas alimentarias que condicionan el estado nutricional en etapas posteriores de la vida. Si estos hábitos son adecuados, contribuirán a una buena salud en la edad adulta $(11,12)$. La evidencia actual señala que, cuando los niños cursan el cuarto año de enseñanza básica, específicamente a la edad de nueve años, éstos ya han adquirido una mayor autonomía para internalizar los conocimientos y modificar actitudes respecto a los alimentos y además dan una visión intermedia de lo que podría esperarse en términos de comprensión y rendimiento de niños entre tercero a quinto básico (8).

La elevada y creciente prevalencia de sobrepeso y obesidad en los niños chilenos requiere de la identificación de sus causas y de las medidas adecuadas para enfrentarlos, entre las cuales la educación en alimentación y nutrición tienen un papel fundamental (13).

Algunos estudios epidemiológicos muestran que aunque en ocasiones la población está informada y conoce los conceptos básicos de una dieta saludable, estos conocimientos no se traducen en consumos reales de alimentos que formen parte de una dieta equilibrada (12), en definitiva, no se ponen en práctica los conceptos aprendidos (14).

La adquisición de los conocimientos se considera positiva, en la medida que repercute y refuerza la práctica alimentaria correcta creando buenas actitudes hacia la alimentación saludable (15). Sin embargo, no es suficiente que la información sea correcta, es necesario también que se produzca la modificación o abandono de estos hábitos alimentarios insanos y erróneos $(13,16)$.

Por otra parte, el estado nutricional está relacionado con múltiples factores, tales como los ingresos económicos para cubrir las necesidades nutricionales con alimentos adecuados y el nivel educativo de los padres con respecto a la nutrición $(17,18)$ lo que sin un asesoramiento adecuado sobre la alimentación, influye directamente sobre la calidad de vida de los niños(as), y por ende, en la calidad de vida del futuro adulto (19).

La ingesta nutricional inadecuada del grupo familiar, tiene consecuencias importantes debido a que la malnutrición, puede afectar negativamente al desarrollo cognitivo de los niños durante la enseñanza básica (29).

La configuración de los hábitos alimentarios en la edad infantil y juvenil responde a una estructura compleja en la que interactúan múltiples factores. Sin duda, la familia y el medio escolar son dos elementos clave en este sentido (21). Se ha observado el impacto que produce la escuela sobre las prácticas de alimentación de niños participantes en programas de educación nutricional, cuyo éxito parece depender en gran parte de la preparación e interés de los profesores, la flexibilidad y adecuación de los programas de enseñanza y su proyección a nivel de la familia y la comunidad (22).

Una alimentación saludable en el hogar debe complementarse con lo que los niños consumen en la escuela. Aquí la educación alimentaria adquiere un rol esencial; se considera un proceso multidisciplinario, a través de los profesores y profesionales de la salud (23), que involucra la transferencia de información, el desarrollo de actitudes y la modificación de prácticas alimentarias, cuando sea necesario, para promover el buen estado de nutrición y salud. No debe contemplar sólo la adquisición de conocimientos, sino su interpretación significativa y su transformación en acciones para toda la vida (10).

Se ha pasado de considerar la obesidad infantil como un problema individual a caracterizarla como una epidemia. La necesidad de reflexionar de manera colectiva sobre el problema, ha devenido en la necesidad de determinar el nivel de conocimiento de los niños en etapa escolar respecto a hábitos alimentarios y nutrición, debido a que, la escuela se considera como depositario de las responsabilidades sociales de educación y contribuidor de hábitos alimentarios, de forma independiente a los niveles socioeconómicos de los niños.

El objetivo de la presente investigación fue determinar el estado nutricional y los conocimientos de los estudiantes de $4^{\circ}$ año básico, respecto a alimentación y hábitos saludables, en colegios privados y particulares subvencionados de Talca, y la influencia de las calificaciones y gasto en educación en estos resultados.

\section{SUJETOS Y MÉTODOS}

Estudio de enfoque cuantitativo, de tipo observacional correlacional de corte transversal, realizado entre el 15 de noviembre y 14 de diciembre de 2009. La población de referencia consistió en 1401 estudiantes de cuarto año básico matriculados con fecha de 2009 en 29 establecimientos educacionales de la zona urbana perte- 
neciente a la ciudad de Talca, de los cuales 1205 alumnos pertenecen a colegios particulares subvencionados y 196 escolares matriculados en 4 colegios particulares registrados al momento de la investigación.

Los sujetos que formaron parte de la muestra fueron 273 alumnos, determinados con un nivel de confianza del $95 \%$ y un error de muestreo de $5 \%$.

El método de selección de las unidades de observación fue al azar y estratificado según tipo de colegio (particulares y subvencionados). En una primera etapa, se estratificó a la población según tipo de establecimiento y se calculó el tamaño muestral de manera proporcional al tamaño poblacional de cada estrato. Para identificar a los colegios, se recurrió a las listas de directorio de establecimientos educacionales del año 2008 del Ministerio de Educación, y de los alumnos regulares facilitados por cada colegio de la ciudad de Talca. En una segunda etapa, se seleccionó a los cursos (4A-4B-4C, etc.) y estudiantes de manera aleatoria (azar simple, por medio de tabla de números aleatorios generados por el programa Excel 2003) para cada tipo de colegio hasta completar el tamaño de muestra mínimo que se le asignó para cada estrato.

Dado que el tamaño de la población para colegios particulares es menor respecto de quienes cursan sus estudios en establecimientos subvencionados (196 y 1205 , respectivamente), se decidió realizar una estratificación con afijación no proporcional, para contribuir a estabilizar la muestra de los dos grupos y también para evitar el riesgo que la inasistencia o algún otro evento pudiera afectar el tamaño de muestra calculado, se aumentó el número de niños seleccionados de colegios particulares de 34 a 53 (55\% de incremento) y desde 206 hasta 220 (6.80\% de incremento) en colegios subvencionados; de tal modo que la razón de colegios subvencionados y particulares quedó en 4:1.

Los criterios para incluir a las unidades de análisis en este estudio fueron pertenecer como alumno matriculado en los colegios particulares y particulares subvencionados de la ciudad de Talca, de ambos sexos, que al momento de la recolección de información estén cursando el cuarto año básico. Se excluyeron a estudiantes que hayan repetido algún curso anterior al cuarto año básico de enseñanza o con algún tipo de trastorno mental o psicológico, tales como síndrome de Down, autismo, depresión endógena, entre otros.

El nivel de conocimientos de los alumnos según los aprendizajes esperados incluidos en la asignatura Comprensión del Medio, se recolectó mediante un instrumento construido para este objetivo, el cual contó con toda la información alimentaria que existe con respecto a un niño de nueve años de edad, a través de preguntas dirigidas a éstos, las cuales fueron construidas siguiendo las Guías Alimentarias del Ministerio de Salud y fueron contextualizadas en la alimentación que debe llevar un niño con la edad de un estudiante de cuarto año básico. Las preguntas fueron agrupadas en tres ítems: 1) nutrición general (siete preguntas), 2) alimentación saludable (diecisiete preguntas) y 3 ) estado nutricional y actividad física (dos preguntas). Tanto el ítem uno como el tres se ponderaron con el mismo porcentaje (30\%). Al ítem dos se le asignó un 40\%. La duración del instrumento aplicado fue de 15 minutos por alumno.

Se calificó un conocimiento aceptable, si el porcentaje de respuestas correctas del instrumento fue igual o superior al 75\%, respecto del total de los ítems.

El instrumento fue sometido a una validación de contenido o juicio de expertos ( 3 nutricionistas y 1 docente de enseñanza básica) y para medir la confiabilidad del instrumento, se recurrió al coeficiente $\alpha$-Cronbach.

Con el objetivo de describir el estado nutricional de la muestra, se midió el peso y talla, para ello se utilizó una balanza mecánica con tallímetro incorporado, con precisión mínima de $0,5 \mathrm{~cm}$ marca Detecto, modelo 2391, con una capacidad de peso 180 kilos y capacidad de talla de 2 metros.

Las mediciones antropométricas se realizaron utilizando las técnicas propuestas por la Organización Mundial de la Salud (OMS) (24).

La información respecto al rendimiento escolar y mensualidad del establecimiento (medido en moneda nacional), se obtuvieron mediante información del profesor jefe, docente coordinador de enseñanza básica y libro de clases (registro de calificaciones general obtenida por los estudiantes del año en curso, hasta el momento del trabajo de campo).

Para poder aplicar el instrumento a los niños y posteriormente realizar la toma de su peso y talla, se elaboró un consentimiento Informado dirigido a los padres.

Se utilizó el programa EPI INFO 2002 (referencia CDC-NCHS) para la obtención de las variables IMC (Percentil) y edad (a partir de las fechas de nacimiento y medición), donde se determinó el estado nutricional utilizando los criterios de: bajo peso; percentil menor a 10, normal; percentil 10 a 85, riesgo de obesidad; percentil 85 a 95 y Obesidad; percentil mayor a 95, de las referencias propuestas por el Center for Disease Control de EEUU (CDC), aceptadas por el Ministerio de Salud de Chile (25).

Luego del trabajo de campo, se construyó una matriz de datos en el programa Microsoft Excel versión 2003 para Windows, el cual fue exportado al software SPSS versión 15,0 para su análisis.

La descripción de los datos se efectuó mediante 
medidas de tendencia central y de dispersión, así como también frecuencias absolutas y relativas para aquellas variables de tipo ordinal o nominal. Con el fin de comparar medias de dos grupos se utilizó la prueba de t-Student, coeficiente de correlación de Pearson y test Ji (2). El nivel de significación usado para realizar las inferencias fue de 0,05 .

\section{RESULTADOS}

De los 273 escolares estudiados, el 80,6\% de la muestra provino de colegios particulares subvencionados $(n=220)$, de los cuales 88 son niñas $(40,0 \%)$. De los estudiantes de colegios particulares (19,4\% de la muestra), el $47,17 \%$ de ellos son niños. No se observó una diferencia significativa entre los grupos de niños y niñas respecto al tipo de colegio donde se extrajo la muestra (Test Exacto de Fisher: p=0,062). Es decir, tanto las niñas como los niños se reparten homogéneamente en los dos tipos de establecimientos educacionales.

El 51,3\% de los estudiantes pertenecientes a colegios particulares subvencionados presentaron un estado nutricional normal $(n=113)$, porcentaje similar al observado en el grupo de niños provenientes de colegios particulares $(50,9 \% ; n=27)$. (tabla 1$)$

La confiabilidad del instrumento utilizado, obtenida a través del cálculo del test $\alpha$-Cronbach, arrojo un valor de 0,7715 ( $n=273)$, considerado como una consistencia interna aceptable $(0,70$ y 0,80$)$, lo que es indicativo que sus mediciones son comparativamente estables y consistentes para evaluar las variables de interés.

El porcentaje medio obtenido por los estudiantes respecto al test de conocimientos de hábitos alimentarios

\section{TABLA 1}

Estado nutricional según tipo de colegio de donde provienen los estudiantes de cuarto año básico. (n=273)

\begin{tabular}{|lccccc}
\hline TIPO DE COLEGIO & $\begin{array}{c}\text { Eajo Peso } \\
\mathbf{n}(\%)\end{array}$ & $\begin{array}{c}\text { Normal } \\
\mathbf{n}(\%)\end{array}$ & $\begin{array}{c}\text { Sobrepeso } \\
\mathbf{n}(\%)\end{array}$ & $\begin{array}{c}\text { Obesidad } \\
\mathbf{n}(\%)\end{array}$ & TOTAL \\
\hline Particular subvencionado & 0 & $113(51,3)$ & $62(28,2)$ & $45(20,5)$ & $220(100,0)$ \\
Particular & $1(1,9)$ & $27(50,9)$ & $17(32,1)$ & $8(15,1)$ & $53(100,0)$ \\
TOTAL & $1(0,4)$ & $140(51,3)$ & $79(28,9)$ & $53(19,4)$ & $273(100,0)$ \\
\hline Asociación lineal por lineal. Ji2 $=0,525(1 \mathrm{gl}), \mathrm{p}=0,469$ & & & &
\end{tabular}

\section{TABLA 2}

Rendimiento académico y puntaje obtenido en el test de conocimientos en alimentación saludable y hábitos alimentarios por estudiantes de cuarto año básico según tipo de colegio. (n=273)

\begin{tabular}{|c|c|c|c|c|c|c|}
\hline \multirow[t]{2}{*}{ RENDIMIENTO } & \multirow[t]{2}{*}{ TOTAL } & \multirow[t]{2}{*}{ Mín } & \multirow[t]{2}{*}{ Máx } & \multicolumn{2}{|c|}{ Tipo de Colegio } & \multirow[t]{2}{*}{$\mathbf{P} *$} \\
\hline & & & & $\begin{array}{l}\text { Particular } \\
\text { subvencionado } \\
\quad(\mathbf{n}=\mathbf{2 2 0})\end{array}$ & $\begin{array}{c}\text { Particular } \\
(n=53)\end{array}$ & \\
\hline $\begin{array}{l}\text { Nota Asignaturas } \\
\text { del Currículo }\end{array}$ & $5,75 \pm 0,69$ & 3,5 & 6,9 & $5,68+0,70$ & $6,04+0,57$ & 0,001 \\
\hline Puntaje total $(100 \%)$ & $61,47 \pm 14,75$ & 11,34 & 92,94 & $59,84 \pm 14,26$ & $68,23 \pm 14,98$ & $<0,001$ \\
\hline Ítem 1 (30\% del total) & $17,14 \pm 5,09$ & 4,29 & 30,00 & $16,54 \pm 4,98$ & $19,65 \pm 4,86$ & $<0,001$ \\
\hline Ítem 2 (40\% del total) & $23,78 \pm 5,56$ & 7,06 & 37,65 & $23,33 \pm 5,49$ & $25,66 \pm 5,54$ & 0,006 \\
\hline Ítem 3 (30\% del total) & $20,55 \pm 9,61$ & 0,00 & 30,00 & $19,98 \pm 9,76$ & $22,92 \pm 8,63$ & 0,045 \\
\hline
\end{tabular}


fue de $61.5 \%$, siendo mayor en los particulares $(p<0,01)$. Se destaca que en el ítem II "alimentación saludable" ningún niño logró obtener el puntaje máximo. En cuanto al porcentaje de conocimiento alcanzado por los niños según tipo de colegio, existe una diferencia significativa, siendo los de establecimiento particular superior $(\mathrm{p}<0,05)$. (tabla 2)

Se analizó la influencia del promedio de notas (escala de 1 a 7) en los conocimientos alimentarios de los niños. Se observó que en los colegios particulares subvencionados la variabilidad del conocimiento en alimentación saludable y hábitos alimentarios de los niños se explica en 19,2\% por la variabilidad del promedio de notas, siendo esta relación estadísticamente significativa $(\mathrm{r}=0.442 ; \mathrm{p}<0.001)$ (tabla 3) (gráfico1).

La evidencia indica que el aumento en una décima

\section{TABLA 3}

Correlación del rendimiento académico y puntaje obtenido en el test de conocimientos en alimentación saludable y hábitos alimentarios por estudiantes de cuarto año básico según tipo de colegio. (n=273)

\begin{tabular}{llllll}
\hline TIPOS DE COLEGIOS & VARIABLES & B & Error típico & t & P \\
\hline Colegios particulares & Constante & 8,728 & 7,077 & 1,233 & 0,219 \\
-subvencionados & Promedio de notas & 8,992 & 1,236 & 7,277 & $<0,001$
\end{tabular}

$\mathrm{R}=0,442 ; \mathrm{R}^{2}=0,192(p<0,001)[\hat{Y}=8,728+8,992 \cdot$ nota $]$

$\begin{array}{llccrr}\text { Colegio particulares } & \text { Constante } & 32,775 & 21,560 & 1,520 & 0,135 \\ & \text { Promedio de notas } & 5,869 & 3,553 & 1,652 & 0,105\end{array}$

$\mathrm{R}=0,225 ; \mathrm{R}^{2}=0,051(\mathrm{p}=0,052)[\hat{Y}=32,775+5,869 \cdot$ nota $]$

Variable de estudio: Puntaje total de los estudiantes respecto a conocimientos de hábitos alimentarios y nutrición.

\section{GRÁFICO 1}

Puntaje total del test de conocimientos en alimentación saludable y hábitos alimentarios con el promedio de notas según tipo de colegio $(n=273)$.

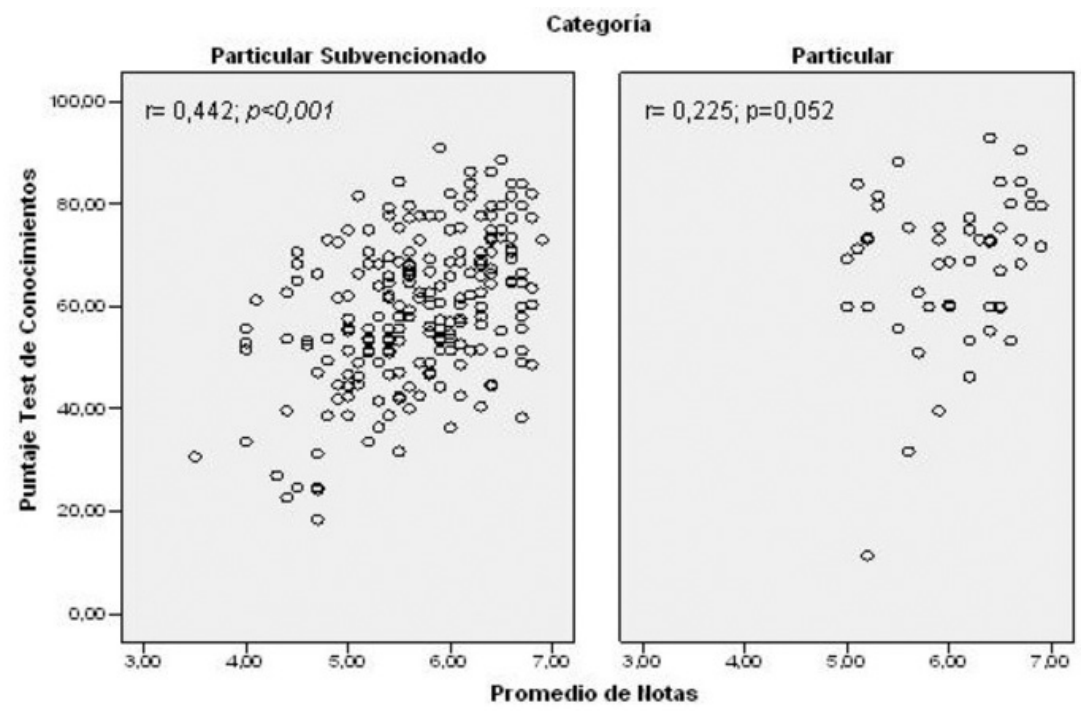


en el rendimiento escolar, medido a través del promedio de notas, aumenta en $9 \%$ el conocimiento referente en hábitos alimentarios de los escolares estudiados (coeficiente $\beta=8.992$ ). En el caso de los estudiantes de colegios particulares (tabla 3 ) tales variables no se correlacionan significativamente $(p>0.05)$, dado que la variabilidad del promedio de notas sólo explica un $5.1 \%$ de la variabilidad del conocimiento respecto de los hábitos alimentarios $(\mathrm{R} 2=0.051)$.

El gráfico 2 muestra diferencias significativas en los puntajes obtenidos por los niños del estudio respecto a las mensualidades que sus padres y/o apoderados pagan para su educación $(\mathrm{p}<0,001)$. Al comparar niños que no pagan respecto del resto se observó una diferencia en el puntaje de conocimientos de entre 6,6 y 14 puntos, aproximadamente $(\mathrm{p}<0,05)$, observándose que a mayor brecha del valor de mensualidad, mayor es la diferencia en el puntaje promedio relacionado con sus conocimientos alimentarios.

Los apoderados que logran pagar entre $\$ 15.000$ y
$\$ 30.000$ pesos respecto a niños que tienen una mensualidad menor a $\$ 15.000$ pesos, no se observó diferencia significativa en el puntaje obtenido. Sin embargo, al comparar el grupo de niños, en los cuales sus familias logran pagar entre 15 y 30 mil pesos respecto de quienes cancelan una cifra superior a $\$ 100.000$, se observó una diferencia significativa a favor de estos últimos, anotando en promedio 6,6 puntos más en el test de conocimientos alimentarios $(\mathrm{p}<0,001)$.

\section{DISCUSIÓN}

En lo que respecta al estado nutricional de los niños que cursan primer ciclo de formación básica (NB1 y NB2) (26), que abarcan niños entre el primer y cuarto año básico, los porcentajes de malnutrición por exceso de los estudiantes de $4^{\circ}$ año evaluados se asemejan a escolares de 6 años que ingresaron a primer año básico en 2004, presentando una prevalencia de obesidad de $17,3 \%$, aunque el indicador peso/talla $>2$ proviene de la NCHS, patrón distinto al usado en este trabajo, que usó

\section{GRÁFICO 2}

Puntaje obtenido en el test de conocimientos de alimentación y nutrición según arancel mensual de los establecimientos educacionales en estudio. $(n=273)$

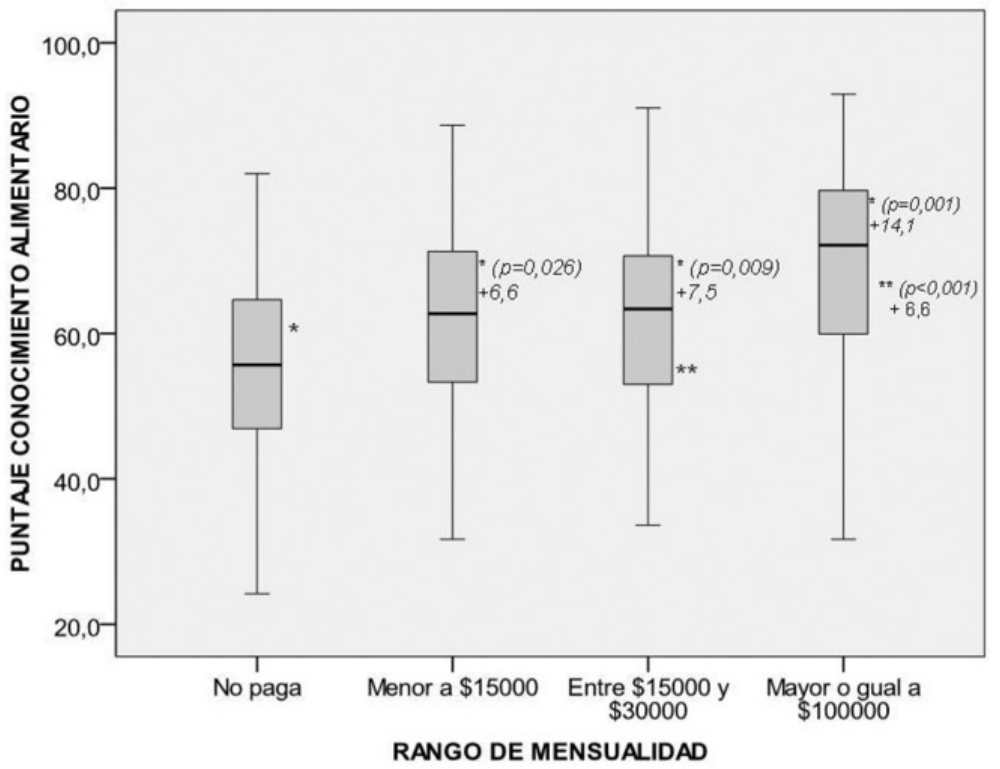

ANOVA. $\mathrm{F}=10,766, \mathrm{p}<0,001$ (Test de Homogeneidad de Levene: 0,332; $\mathrm{p}=0,802$ )

Comparaciones múltiples (Test de Tukey) del Puntaje Total según mensualidades (diferencias promedio entre dos mensualidades significativas).

* Comparación entre estudiantes de establecimientos educacionales que no pagan mensualidad respecto del resto de los tramos.

** Comparación entre estudiantes cuyas familias pagan entre \$15000 y \$30000 respecto de quienes pagan mensualidades superiores a $\$ 100000$ pesos. 
la norma OMS la cual tiende a diagnosticar más personas con malnutrición por exceso). En preescolares asistentes a la Junta Nacional de Jardines Infantiles (JUNJI), esta cifra fue de 10,6\% en 2005 (Según el estudio de Amigo et al, la variabilidad del IMC se explica casi exclusivamente por factores relacionados con la/el niña/o y su familia, en tanto la escuela tiene un aporte no significativo (28), es por esto que la escuela debe transformarse en el ente principal de creación de hábitos saludables en los niños y niñas fomentando la alimentación saludables y la actividad física, además de buenas prácticas de vida.

Se demuestra en este estudio que no existe un adecuado conocimiento en lo que respecta a hábitos y alimentación saludable en los niños, ya que la muestra en estudio no logró superar el $75 \%$ de los conocimientos esperados, tanto en colegios particulares como particulares subvencionados, situación similar a la observada en 249 estudiantes chilenos que egresaban de la educación básica en el Área Metropolitana de Chile, la cual evaluó contenidos relacionados con alimentación, requerimientos, higiene alimentaria, personal y ambiental, donde se obtuvo que el $39.8 \%$ de los alumnos obtuvo conocimientos suficientes (29). Los ítems que presentaron un menor puntaje fueron nutrición básica y alimentación saludable, Pinto et al explica este fenómeno a que estas áreas son de baja relevancia en las planificaciones académicas de los profesores y por otra parte, son temas que habitualmente para los docentes no son de gran interés (30).

La mensualidad demostró ser importante en la adquisición de conocimientos alimentarios nutricionales. Los hijos de familias que pueden cancelar más por la educación de sus hijos logran una mejor calidad de aprendizaje. Diversos estudios señalan similares características, las desigualdades sociales son la piedra angular de las diferencias en educación. Igualmente esta demostrado que el nivel socioeconómico se asocia con el estado nutricional. Niños de NSE bajo tienen una mayor prevalencia de malnutrición por exceso, conclusión reafirmada por Liberona et al (31).

Los niños que ingresan al colegio con problemas de exceso de peso, no logran modificar sus costumbres, conductas o hábitos, adquiridos con anterioridad, durante el transcurso de la etapa escolar (32), por lo que no es de gran impacto describir que los conocimientos en hábitos y conductas alimentarias de los niños son escasas. Lo anterior se explica, según Olivares (33), al hacer referencia que en la escuela no existen acciones que modifiquen hábitos de alimentación y actividad física adquiridos y mantenidos en el hogar, o que no se realizan acciones apropiadas para cambiar dichos hábitos, por lo que es urgente la amalgama entre la escuela y los centros de salud como entes que promuevan los determinantes de salud y que con la prevención se ataquen en conjunto los condicionantes.

Al comprobar que, al menos hasta cuarto año básico, la escuela no está cumpliendo un papel definido como elemento preventivo o controlador del exceso de peso, es aún más difícil que otros organismos cumplan un papel protector, ya que el vehículo más cercano: la escuela, no consigue jugar un rol contrarrestador de los comportamientos familiares e individuales.

Por otro lado, la evidencia de este estudio señala que los niños pertenecientes a ambos tipos de colegios, requieren intervenciones educativas orientadas a mejorar su alimentación y aumentar la actividad física para alcanzar un mejor estado nutricional. Sin embargo, se reconoce que las intervenciones educativas por sí solas no lograrán los cambios requeridos si los escolares no cuentan con ambientes apropiados y el apoyo necesario para llevar a la práctica las conductas saludables en el hogar, la escuela y la comunidad (34).

Debido a la relación entre la malnutrición por exceso y las enfermedades crónicas no transmisibles del adulto, surge la necesidad de implementar estrategias educacionales en alimentación y nutrición tanto para estudiantes como para los docentes. Estos programas se deben iniciar a edad temprana, orientándose hacia la promoción de un estilo de vida más saludable, con especial énfasis en la alimentación y la actividad física, así como en la prevención de la obesidad y las enfermedades crónicas asociadas (35).

\section{RESUMEN}

La malnutrición por exceso en la etapa escolar es un problema que también se puede abordar en el aula, dado que los niños pasan gran parte del tiempo en el establecimiento educacional. Se estudiaron los conocimientos de alimentación de alumnos de cuarto año básico de colegios particulares y particulares subvencionados de la ciudad de Talca. Se creó y aplicó un instrumento para medir los conocimientos de hábitos en alimentación para niños en una muestra de 273 escolares. En promedio, el porcentaje de conocimientos que poseen los estudiantes fue de $61,4 \pm 14,75 \%$. Los alumnos de colegios particulares tuvieron un mejor dominio en conocimientos sobre alimentación $(\mathrm{p}<0.05)$. El conocimiento insuficiente podría repercutir en el estado nutricional de los niños. Este tema se debe afrontar desde una perspectiva multifactorial, ya que sólo los conocimientos no son indicador de éxito suficiente en la recuperación nutricional de los niños.

Palabras clave: Conocimiento, hábitos alimentarios, escolares, cuarto año básico, malnutrición por exceso, subvención escolar. 
Dirigir la correspondencia a:

Sr. José Luis Pino Villalón

Director Carrera Nutrición y Dietética

Universidad del Mar,

sede Centro Sur

4 Poniente 1223

Talca, Chile

Fono: (71) 417000

Fax: (71) 417062

E-mail: jose.pino@udelmar.cl

\section{BIBLIOGRAFÍA}

1. Moraga F, Rebollo M.J, Borquéz P, Cáceres J, Castillo-Duran C. Tratamiento de la obesidad infantil: Factores pronósticos asociados a una respuesta favorable. Rev Chil Pediatr. 2003; 74: 374-80.

2. Crovetto M, Mirta, Vio Del R, Fernando. Antecedentes Internacionales y Nacionales de la Promoción de Salud en Chile: Lecciones Aprendidas y Proyecciones Futuras. Rev Chil Nutr. 2009, 36 (1):32-45

3. Martínez Rubio, Ana. Aplicación del modelo de Planes Integrales de Salud para el abordaje de la Obesidad Infantil de Andalucía. XXIV. Jornada de Pediatría de Gipuzkoa. Gipuzkoako XXIV. Pediatría Jardunaldia. Asociación Vasca de Pediatría de Atención Primaria, oct.2007, Dمnمnstia-San Sehastián Disponibleen htth-/wwwavpaporg/documentos/ gipuzkoa2007/plansaludobs.pdf, [Consultado el 26 de junio de 2010]

4. Ceinos Arcones M, Junco Torres I, Garcia Crespo P. Hábitos Alimentarios Saludables. Educación para la Salud. Nutr Clin Diet Hosp 2008; 28(supl. 1) 44.

5. Rodríguez O Lorena. Situación nutricional del escolar y adolescente en Chile. Rev Chil Pediatr 2007, 78 (5):523-33.

6. Caballero C, Beltran B, Del Pozo S. Hábitos Alimentarios en Escolares de Villanueva de la Cañada (Madrid). Diferencias entre niñas y niños. Nutr Clin Diet Hosp 2008; 28 (supl. 1), 69.

7. Olivares Sonia, Yáñez Rossana, Díaz Nora. Publicidad de alimentos y conductas alimentarias en escolares de $5^{\circ}$ a $8^{\circ}$ básico. Rev Chil Nutr. 2003; 30(1): 36-42.

8. Kain B, Juliana, Olivares C, Sonia, Castillo A, Marcela, Vio D, Fernando. Validación y aplicación de instrumentos para evaluar intervenciones educativas en obesidad de escolares. Rev Chil Pediatr. 2001, 72 (4): 308-18.

9. Kain J, Albala C, Garcia F, Andrade M. Obesidad en el preescolar: Evolución antropométrica y determinantes socioeconómicos. Rev Méd Chil. 1998; 126: 271-278.
10. Portal Educativo. Publicación de la dirección regional de cultura y educación de la provincia de Buenos Aires, Año 2, $\mathrm{N}^{\circ} 4$ Septiembre/Octubre 2007 Disponible en h http //ahe gov ar/lainstitlcion/revistacomponents/revista/archivos/portalducativo/numerol/archivosparaimprimir/74aalimentacionylaescuela.pdf [Copnsultado el 26 de junio de 2010]

11. Montero Bravo A, Ubeda Martin N, Garcia Gonzalez, A. Evaluación de los hábitos alimentarios de una población de estudiantes universitarios en relación con sus conocimientos nutricionales. Nutr Hosp 2006, 21(4):466-73.

12. Ivanovic D, Álvarez ML, Truffello I. Conocimientos y hábitos alimentarios y nutricionales de estudiantes que egresan de educación básica en el área Metropolitana de Santiago, Chile (Food and nutrition knowledge of students graduating from basic education in the Metropolitan Area of Santiago, Chile). Arch Latinoam Nutr. 1986; 36: 152-65.

13. Vio Del R, Fernando, Salinas C, Judith. Promoción de salud y calidad de vida en chile: una política con nuevos desafíos. Rev Chil Nutr. 2006, 33(SS 1): 252-9.

14. Monteiro CA, Conde WL, Lu B, Popkin B. M. Obesity and inequities in health in the developing world. Int J Obes Relat Metab Disord. 2004; 28(9): 1181-6.

15. Benavides R, Martha L, Bermúdez V, Sayra Y. Estado nutricional en niños del tercer nivel de los preescolares: El Jardín de Infancia Rubén Darío y Escuela Rubén Darío de la ciudad de León. Universitas, (C) 2008 UNAN-León, Editorial Universitaria $2008,2(2) \cdot 5-12$ Disponible en $\cdot$ http.//universitas unanleon.edu.ni/pdf/volumen2_nro2/arto1.pdf Consultado el 3 de julio de 2010]

16. Montero Bravo, A.; Ubeda Martin, N. Y Garcia Gonzalez, A. Evaluación de los hábitos alimentarios de una población de estudiantes universitarios en relación con sus conocimientos nutricionales. Nutr Hosp 2006, 21(4): 466-73.

17. Zaini M. Z, Lim C. T, Low W. Y, Harun F. Factors affecting nutritional status of Malaysian primary school children. Asia Pac J Public Health. 2005; 17 (2): 71-80.

18. Zacarías H Isabel, Pizarro Q, Tito, Rodríguez O, Lorena, González A., Daniela, Domper R., Alejandra. Programa "5 al día" para promover el consumo de verduras y frutas en chile. Rev Chil Nutr. 2006, 33 (Suppl 1): 276-80.

19. Ministerio de Salud, Chile, Guía de Alimentación del Niño(a) Menor de 2 años, Guía de Alimentación 
hasta la Adolescencia, 2005, Disponible en . http./4 oww redsalud oovel/archivos/alimentosynutricion estrategiaintervencion/Guia_guia_adolescencia. pdf, [Consultado el 03 de julio de 2010].

20. Galal OM, Ismail I, Gohar A S, Foster Z. Schoolteachers' awareness about scholastic performance and nutritional status of Egyptian schoolchildren. Food Nutr Bull 2005, 26 (2 Suppl 2): S275-80.

21. Aranceta Bartrina $\mathbf{J}$, et al. Hábitos alimentarios de los alumnos usuarios de comedores escolares en España. Estudio «Dime Cómo Comes». Aten Primaria 2004; 33(3):131-9.

22. Olivares $\mathrm{C}$, Sonia et al. Antecedentes para la enseñanza de salud en el sistema escolar chileno. Educ Méd Salud 1983; 17 (2); 163-72.

23. Carlson E, Kipps M, Thomson J. Influences on the food habits of some ethnic minorities in the United Kingdom. Hum Nutr Appl Nutr. 1984, 38(2): 85-98.

24. World Health Organization, (OMS), Physical Status: The Use and Interpretation of Anthropometry, Report of a WHO Expert Committee Technical Report Series, No 854, Ginebra 1995.

25. Ministerio de Salud, Chile. Norma Técnica de Evaluación Nutricional de niños y Niñas de 6 a 18 años. 2003, Disponible en: http://www.redsalud. gov.cl/archivos/alimentosynutricion/estrategiaintervencion/NormaEvNut6a18anos.pdf [Consultado el 03 de julio de 2010].

26. Ministerio de Educación. Chile: http://www.curriculum-mineduc.cl/ [Consultado el 07 de septiembre de 2010].

27. Kain B Juliana, Lera M Lydia, Rojas P Juanita, Uauy D, Ricardo. Obesidad en preescolares de la Región Metropolitana de Chile. Rev Méd Chil. 2007, 135(1): 63-70.

28. Amigo Hugo, Bustos Patricia, Erazo Marcia, Cum- sille Patricio, Silva, Claudio. Factores Determinantes del exceso de peso en escolares: Un estudio multinivel. Rev Méd Chil. 2007; 135: 1510-18.

29. Ivanovic Daniza, Alvarez María de la Luz, Trufello Irene. Conocimientos alimentarios y nutricionales de estudiantes que egresan de educación básica en el area metropolitana de Santiago, Chile / Food and nutrition knowledge of students graduating from basic education in the Metropolitan area of Santiago, Chile. Arch Latinoam Nutr. 1986; 36(1):152-65.

30. Pinto Arturo, Cárdenas Zenobio, Hermosilla Víctor. Conocimientos alimentario-nutricionales de profesores de educación básica de Chiloé, Chile. Rev Chil Nutr. 1985; 13(3):175-80.

31. Liberona Z, Yéssica Engler T, Valerie Castillo V, Oscar Villarroel Del P Luis, Rozowski N Jaime. Ingesta de macronutrientes y prevalencia de malnutrición por exceso en escolares de $5^{\circ}$ y $6^{\circ}$ básico de distinto nivel socioeconómico de la región metropolitana. Rev Chil Nutr. 2008.35 (3): 190-9.

32. Kain J, Albala C, Garcia F, Andrade M. Obesidad en el preescolar: evolución antropométrica y determinantes socioeconómicos. Rev Méd Chil. 1998; 126: $271-8$

33. Olivares $\mathrm{C}$, Sonia et al. Actitudes y prácticas sobre alimentación y actividad física en niños obesos y sus madres en santiago, chile. Rev Chil Nutr. 2006, 33(2):170-9.

34. Olivares C Sonia, Bustos Z Nelly, Lera M Lydia, Zelada María Eugenia. Estado nutricional, consumo de alimentos y actividad física en escolares mujeres de diferente nivel socioeconómico de Santiago de Chile. Rev Méd Chil 2007, 135 (1): 71-8.

35. Araya L, Héctor et al. Prioridades de intervención en alimentación y nutrición en Chile. Rev Chil Nutr. 2006, 33(3):458-63. 